\title{
Development of an online food database for the Batwa and Bakiga communities living in south-western Uganda
}

\author{
G. Scarpa ${ }^{1-2}$, L. Berrang-Ford ${ }^{1-4}$, A. O. Bawajeeh ${ }^{2-3}$, S. Twesigomwe ${ }^{4}$, P. Kakwangire ${ }^{4}$, \\ S. Beer ${ }^{5}$, G. Williams, C. Zavaleta-Cortijo ${ }^{6}$, S. Lwasa $^{7-4}$, R. Peters ${ }^{8}$, H. Rippin ${ }^{9}$ and J. E. Cade ${ }^{2}$ \\ ${ }^{1}$ School of Environment, University of Leeds, LS2 9JT, \\ ${ }^{2}$ School of Food Science and Nutrition, University of Leeds, LS2 9JT, \\ ${ }^{3}$ Department of Food and Nutrition, Faculty of Human Sciences and Design, King Abdulaziz University, Jeddah 3270, \\ Saudi Arabia, \\ ${ }^{4}$ Indigenous Health Adaptation to Climate Change Research Team, \\ ${ }^{5}$ Dietary Assessment Ltd, UK, \\ ${ }^{6}$ Facultad de Salud Publica y Administracion, Universidad Peruana Cayetano Heredia, Peru, \\ ${ }^{7}$ Department of Geography, Makerere University, Kampala, Uganda, \\ ${ }^{8}$ Leeds Institute of Health Sciences, University of Leeds, LS2 9JT and \\ ${ }^{9}$ WHO European Office for Prevention and Control of Non-communicable Diseases (NCD Office) Moscow, Russian
} Federation

Locally relevant food composition data are essential for measuring individual food intake ${ }^{(1)}$. Many developing regions of the world lack this data, especially Indigenous communities, and have to use other national food composition tables as a proxy ${ }^{(2)}$. Our aim was to construct an online database of locally consumed foods for two vulnerable populations living in south-western Uganda.

Using a community-based approach and collaboration with local nutritionists, we collected a list of foods for inclusion in the database through focus group discussions, individual dietary survey and markets and shops assessment. These foods were then processed to create the database using an online dietary assessment tool, myfood $24^{(3,4)}$. The food database was created using 6 steps: identification of foods for inclusion in the database; initial data cleaning and removal of duplicate items; link foods to existing generic food composition tables; mapping and calculation of nutrient content of recipes and foods; quality checks with local and international nutritionists; translation into relevant local languages.

We developed a food composition database for south-western Uganda including 148 commonly consumed foods complete with values for 120 micronutrients and macronutrients. Of the locally reported foods included, $56 \%$ of the items were already available in myfood 24 database, while $25 \%$ were found in the Ugandan, Kenyan, and Tanzania food databases, $18 \%$ came from generated recipes and $1 \%$ from food packaging labels. The food database included $43 \%$ of fruits and vegetables products and dishes, $26 \%$ of cereals items and cereal based dishes (including dishes with higher percentages of cereals than vegetables), 14\% of meat and fish dishes, and $5 \%$ of eggs and diary. Only 3\% of products were sugary or sweet-based and 3\% were included in oils, fats and condiments. Some cooking oils and fats were branded, while the majority of the other food items did not have any brand. Soft drinks (4\%) and alcohol beverages $(1 \%)$ corresponded to $5 \%$ of the foods included in the database.

The tool will be used for assessing the food intake, and tracking under-nutrition among the communities living in Kanungu District, Uganda.

\section{Acknowledgments}

We are very thankful to the Batwa and Bakiga communities that gave us precious information for developing the food database.

\section{References}

1. Kigutha HN (1997) Am J Clin Nutr 65, 1168s-1172s.

2. Rippin HL, Hutchinson J, Evans CEL et al. (2018) Food Nutr Res 62.

3. Carter MC, Albar SA, Morris MA et al. (2015) Nutrients 7, 4016-4032.

4. Albar SA, Carter MC, Alwan NA et al. (2015) BMC Nutr 1, 29. 